\title{
La sal de la vida, vida en la sal: Producción de sal en paisajes de alto valor ecológico \\ The salt of life, life in salt: Salt production in high value landscapes
}

Katia Hueso Kortekaas, Universidad Pontificia de Comillas

DOI: $10.20868 /$ ciur.2020.129.4405

\section{DESCRIPTORES:}

Salinas / sal artesanal / biodiversidad / gourmet / conservación

\section{KEY WORDS:}

Salt works / artesanal salt / biodiversity / gourmet / conservation

\section{RESUMEN:}

Pese a que la sal se considera perniciosa para la vida, los paisajes de la sal y, más en concreto las salinas de evaporación solar, constituyen complejos sistemas biológicos de gran relevancia para la producción de sal de calidad. No se trata sólo de la biodiversidad de especies halófilas que pueda habitarla o de las estrategias fisiológicas que la biota de las salinas tenga para sobrevivir a la sal en el ambiente, sino del entramado de relaciones que entre ellos se producen. El delicado equilibrio entre la red trófica de las salinas con la actividad de producción artesanal de sal, hacen que sean paisajes vivos, que garantizan la conservación de sus valores naturales y culturales. La producción artesanal de sal se puede considerar así una actividad agrícola que resulta en un producto de alta calidad y respetuoso con el medio natural.

\section{ABSTRACT:}

Although salt is considered harmful to life, saltscapes and, more specifically, solar evaporation salinas, are complex biological systems of great relevance to the production of quality salt. It is not only a question of the biodiversity of halophilic species that may inhabit them or the physiological strategies that their biota have to survive the salt present in the environment, but also of the network of relationships between them. The delicate balance between the trophic network of the salinas and the artisanal salt production, make them living landscapes, which guarantee the conservation of their natural and cultural values. Artisanal salt production can thus be considered an agricultural activity that results in a high quality product that is respectful of the natural environment.

\footnotetext{
* Katia Hueso Kortekaas, MSc Biología por la Universidad de Leiden (Países Bajos), Especialista en Espacios Naturales Protegidos por la Universidad Autónoma de Madrid, Master en Ingeniería Ambiental por la Fundación EOI y doctora en Sociedad y Cultura por la Universidad de Barcelona. Profesora asociada en la Universidad Pontificia de Comillas / ICAI y coordinadora del Instituto del Patrimonio y los Paisajes de la Sal (IPAISAL). salinasdeinterior@gmail.com ORCID: https://orcid.org/0000-0002-0353-8725 (Katia Hueso Kortekaas)
} 


\section{LOS ECOSISTEMAS SALINOS}

Se suele pensar que la sal no es un elemento amable con la naturaleza, pues se asocia a muerte y destrucción. Se ha utilizado de forma ritual para simbolizar la derrota del enemigo y derramarla se ha considerado una maldición, pues se consideraba que dejaba los terrenos de cultivo infértiles (Fensham, 1962). Sin embargo, la sal es también fuente de vida. Salados son los fluidos corporales y del agua salada surge la vida. Hay quien incluso defiende que, sin la sal, ésta no habría podido aparecer en la Tierra (Yi et al., 2018). De lo que no cabe duda es de que la sal condiciona de manera muy tangible aquellos lugares en los que aparece, en los llamados paisajes de la sal. Lugares en los que, como se verá, hay mucha vida.

Los paisajes de la sal son aquellos espacios en los que la sal es el elemento clave en torno al cual pivotan los hábitats naturales y la actividad humana (Hueso, 2017). Entre ellos se encuentran las salinas de evaporación solar, quizá los más característicos y en los que se va a centrar esta contribución. Existe abundantísima literatura sobre los diferentes grupos taxonómicos presentes en los paisajes de la sal, desde las halobacterias a las plantas y aves que forman parte de estos hábitats y sus redes tróficas. En los paisajes de la sal españoles, los grupos mejor estudiados son los macrófitos, crustáceos y aves, sobre todo en lagunas saladas (Montes \& Martino, 1987), siendo similar a la de otros países mediterráneos, norte de África y de Asia Central. También se ha estudiado en profundidad el crustáceo Artemia sp. (Hontoria \& Amat, 1992), y las investigaciones muestran que las salinas y lagos hipersalinos del interior de la Península Ibérica son un reservorio para la especie autóctona $A$. salina (Fig. 1), amenazada por su congénere invasora $A$. franciscana (Amat et al., 2007; Green et al., 2005).

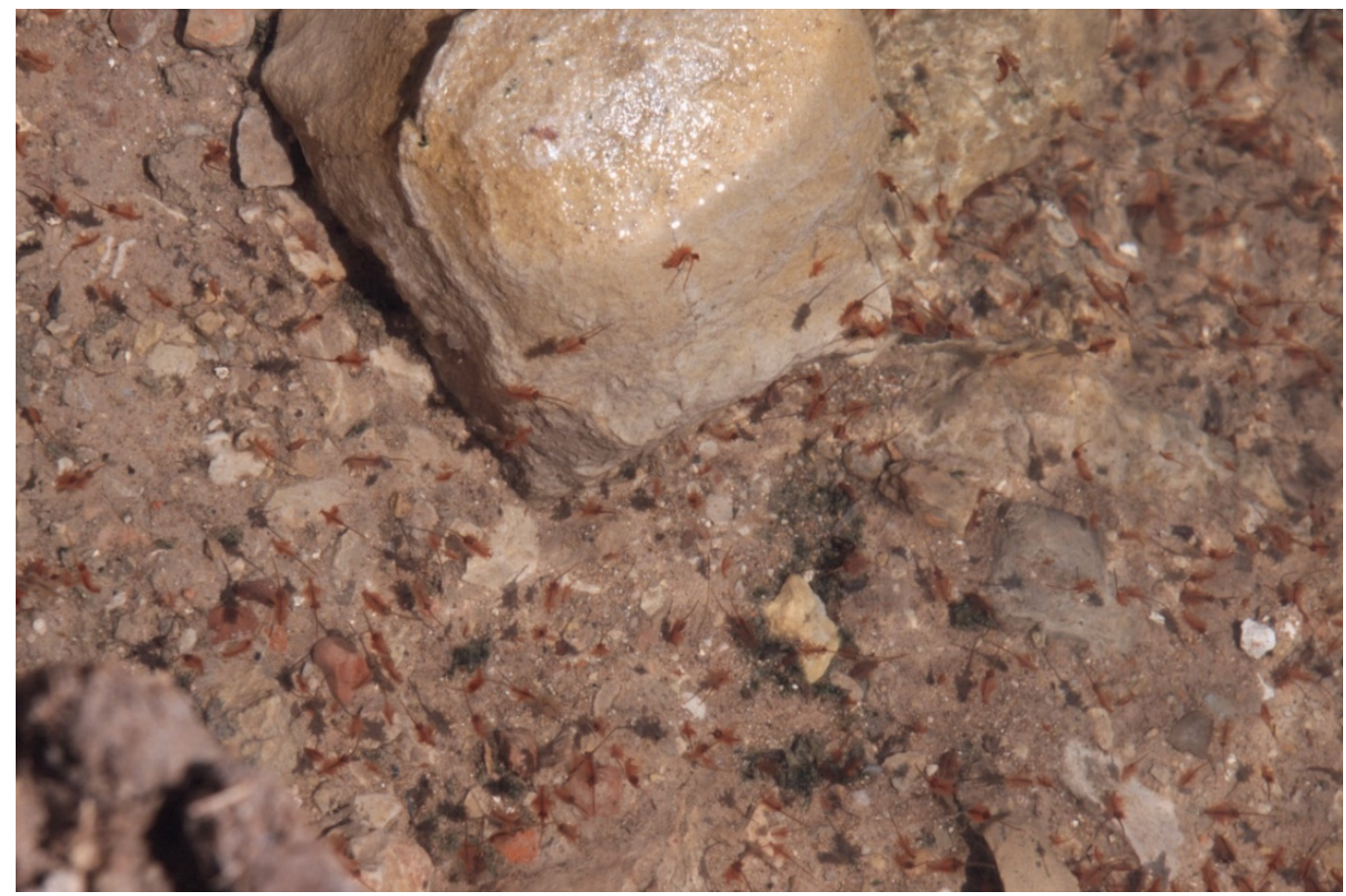

Figura 20: Ejemplares de Artemia salina en las Salinas de San Juan (Guadalajara) 
Fuente: Katia Hueso / IPAISAL

Otra característica interesante de la biota salina ibérica es la presencia de tapetes microbianos. Se trata de comunidades de bacterias que se disponen en capas sobre los fondos de las balsas, formando una alfombra, de ahí el nombre. Para sobrevivir, estos microorganismos requieren ambientes hipersalinos (Guerrero \& de Wit, 1992). Otros investigadores se han centrado en las halobacterias que se encuentran en las salinas y lagos salados de interior (p. ej. Ventosa \& Ventosa, 2004).

Quizá el grupo de fauna más característico de las salinas son las aves, pues en ellas tienen posibilidad de encontrar tanto alimento como refugio. Las salinas constituyen humedales de gran productividad biológica gracias a la relativa sencillez de su red trófica, a su vez causada por la salinidad ambiental. Son además espacios a los que los predadores terrestres no tienen fácil acceso, permitiendo la nidificación de grandes grupos de aves. Las más habituales son las coloniales y las acuáticas, siendo muy típicas de estos ambientes la avoceta (Recurvirostra avosetta), la cigüeñuela (Himantopus himantopus), el chorlitejo patinegro (Charadrius alexandrinus), o especies más vulnerables como el charrancito (Sternula albifrons) o la gaviota de Audouin (Larus audouinii). De entre todas, la más llamativa en las salinas del litoral es el flamenco común (Phoenicopterus roseus) (Carly et al., 2004, López et al., 2010).

Con respecto a la vegetación, las salinas de evaporación solar se caracterizan por presentar orlas de plantas halófilas que se disponen en gradientes de salinidad decreciente, a medida que nos alejamos de la salina. Tendríamos así desde las especies halófilas extremas a especies halotolerantes, hasta llegar a la vegetación generalista dominante, fuera del área de influencia de la sal. Estos gradientes suelen ser más pronunciados en las salinas de interior que los que se encuentran en los ambientes costeros, ya que la salinidad desaparece rápidamente a poca distancia de su origen (manantial, arroyo, lago, etc.). Los paisajes hipersalinos de interior están dominados por especies vegetales típicas de las costas y las marismas costeras, pero son raros y frágiles cuando se encuentran lejos de ellas y a altitudes relativamente altas. Ejemplos de estas especies son Salicornia sp., Suaeda sp., Salsola sp., Arthrocnemum sp., Limonium sp., Glaux maritima, etc. Plantas acuáticas como Ruppia maritima, Chara sp., Riella helicophylla, etc. son también típicas de los humedales hipersalinos, y muchas de ellas están protegidas por leyes europeas y regionales (Montes \& Martino, 1987; Comín \& Alonso, 1988; Casado \& Montes, 1995; Sadoul et al., 1998; Cirujano \& Medina, 2002; Martín et al., 2003; Hueso \& Carrasco, 2008). 


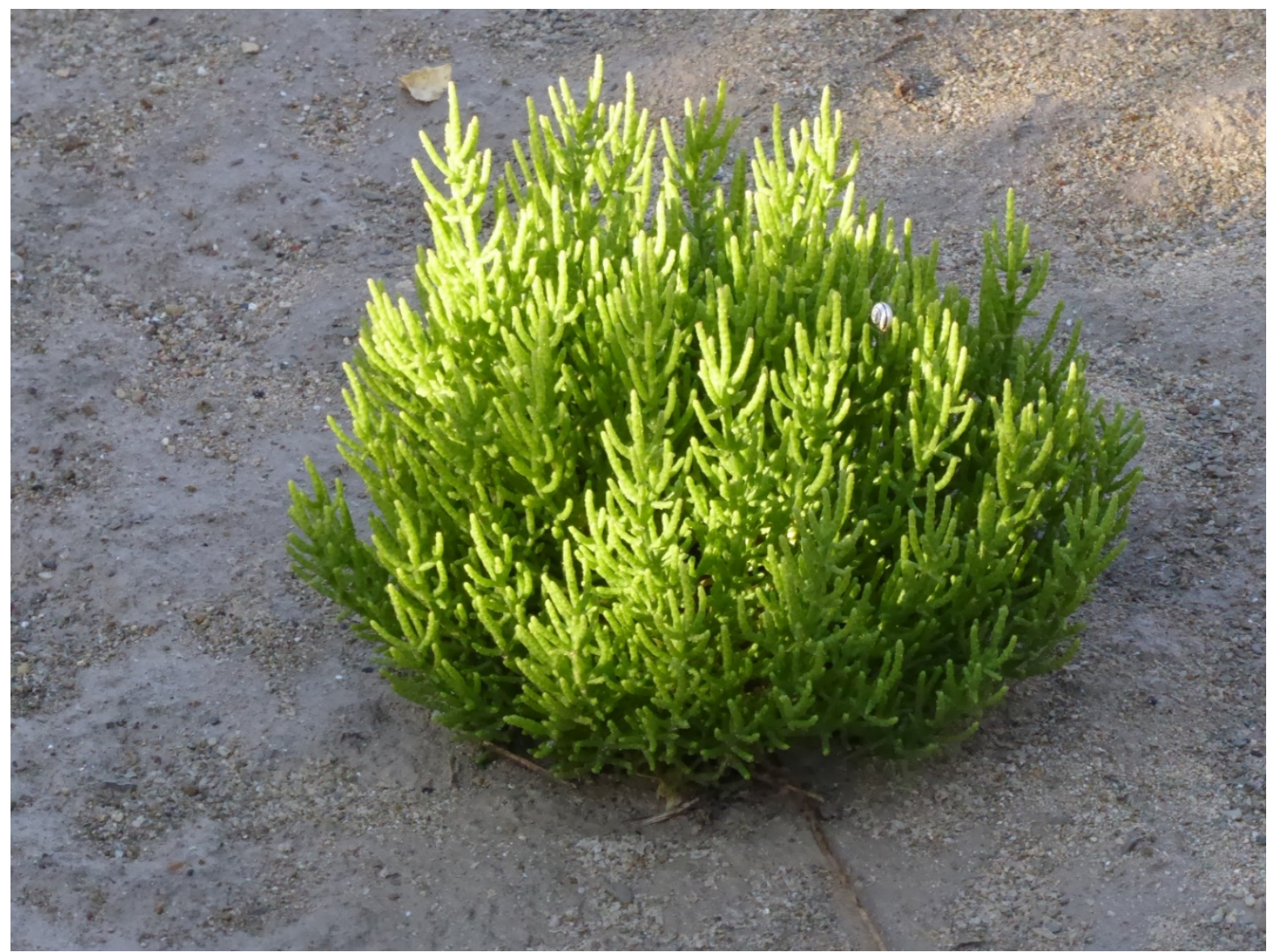

Figura 21: Mata de Salicornia europaea en las salinas de Duernas (Córdoba) Fuente: Katia Hueso / IPAISAL

Como se decía más arriba, el exceso de sal es una condición que pone en peligro la vida de la mayoría de los seres vivos. En condiciones naturales, la biota (es decir, los seres vivos) nativa de los paisajes salados han desarrollado diferentes mecanismos fisiológicos para hacer frente a ella. Algunas plantas secretan sal de sus estructuras aéreas: las suculentas de tallo (las que acumulan sal en los tallos), como el género Salicornia, y las suculentas de hoja (las que acumulan sal en las hojas), como los géneros Suaeda o Salsola. Otras plantas excretan el exceso de sal, como los géneros Limonium, Atriplex o Tamarix y otras la confinan en las vacuolas, unos órganos extracelulares en los que la sal no entra en contacto con las partes vitales de la célula. Otras a su vez protegen sus órganos de la salinidad con ciertas sustancias, como el glicol, un alcohol utilizado habitualmente como anticongelante (Breckle, 2002). Los microorganismos y los pequeños invertebrados utilizan mecanismos similares. Los animales más grandes son poco comunes en los hábitats hipersalinos. El coste fisiológico de desarrollar y mantener estos mecanismos es alto y pocas especies son capaces de hacerlo. Las halófilas, que sí deben invertir en estos mecanismos de defensa, pierden por tanto la capacidad de competir con otras especies cuando se encuentran en condiciones no salinas. Por estas razones, la riqueza y abundancia de especies suele disminuir con la salinidad ( $p$. ej. Moreno et al., 1997, Millán et al., 2002, Gómez et al., 2005). Por otro lado, los organismos halófilos son considerados raros y frágiles, especialmente los que se 
encuentran tierra adentro, en parcelas aisladas de suelo salino rodeadas de hábitats no salinos.

\section{LOS SISTEMAS BIOLÓGICOS DE LAS SALINAS}

Más allá de su biodiversidad y la extraordinaria fisiología de los halófilos, las salinas de evaporación solar son sistemas biológicos complejos que merecen un poco de atención. Consisten en una sucesión de ecosistemas semicerrados y estables, en los cuales cada balsa o conjunto de balsas están en equilibrio y los organismos que las habitan forman una comunidad bien adaptada y establecida (Pedrós-Alió et al., 2000, Dyall-Smith et al., 2003). Cada conjunto de balsas presenta distintas condiciones de profundidad, insolación, salinidad, oxígeno disuelto etc., por lo que constituyen hábitats diferentes entre sí, cada cual con su propia comunidad biológica y red trófica. Sin embargo, son ecosistemas no estancos, pues las balsas están comunicadas secuencialmente unas con otras, por lo que sus redes tróficas están interrelacionadas. Cada organismo vivo presente en las salinas prolifera mejor donde las condiciones son óptimas para él, pero también puede aparecer en otros hábitats, en condiciones subóptimas, participando de la red trófica allí presente.

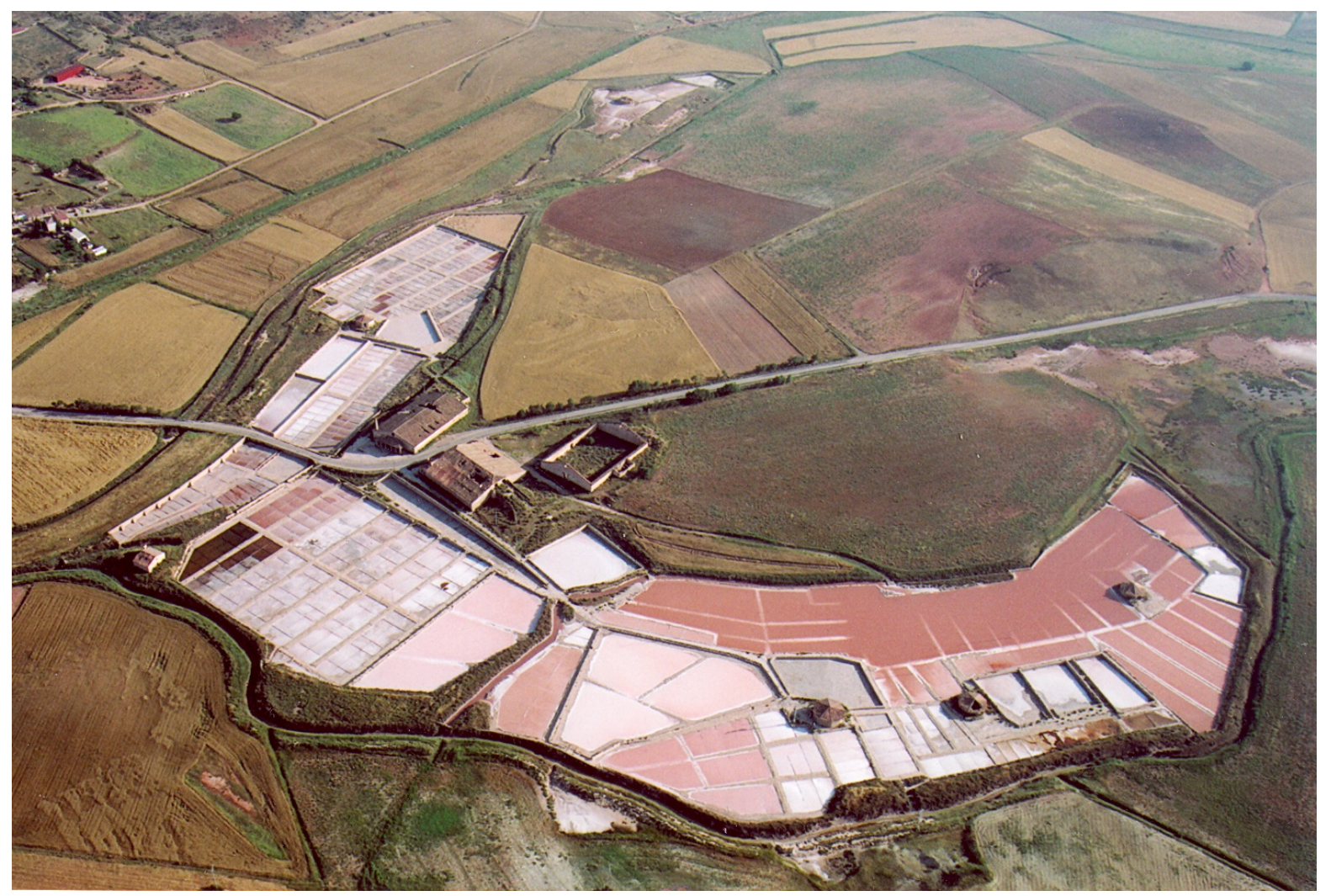

Figura 22: Vista aérea de las salinas de Imón (Gadalajara),

en la que se aprecian balsas teñidas de rojo por la presencia de microorganismos Fuente: Katia Hueso / IPAISAL

La comunidad de una salina se compone de aquellos organismos microscópicos que flotan en el agua (el plancton) y de aquellos que habitan el 
fondo de las balsas (el bentos, que forma los tapetes microbianos referidos más arriba), además de los invertebrados y vertebrados que contribuyen a la red trófica del sistema en su conjunto. Los organismos vivos de una salina son esenciales para la producción de sal, la cual está a su vez íntimamente ligada a las características físico-químicas del sistema (Davis, 1980, 2006). Estos organismos pueden beneficiar o perjudicar la producción de sal, por lo que el conocimiento de su ecología es de extrema importancia para los salineros. Un sistema biológico "en equilibrio" permitirá la obtención de una sal de calidad con un elevado rendimiento, mientras que un sistema "desequilibrado" dificultará la precipitación de sal y sus cristales serán de baja calidad (Davis, 1980, Sundaresan et al., 2006). Así, el plancton contribuye a la producción de sal coloreando la salmuera con un tono rojizo-violáceo que reduce el albedo e incrementa la absorción de energía solar, acelerando la evaporación del agua (Oren \& Dubinsky, 1994). La sal cristaliza así con más rapidez y de ese modo aumenta la productividad de la salina. Mientras, los tapetes microbianos retienen los nutrientes que hay en el agua y sellan el fondo de las balsas de acopio y los arroyos, lo que evita pérdidas de salmuera e infiltraciones de agua dulce y previene la formación de mucílago. Todo ello contribuye a la formación de cristales limpios, grandes y de calidad (Davis, 2000). Los invertebrados contribuyen a su vez a la limpieza de nutrientes y al control de la proliferación de microorganismos, clarificando la salmuera y limpiándola de materia en suspensión. Constituyen además el alimento para las aves, cuyas heces y cadáveres aportan nutrientes de nuevo al sistema, cerrándose así el ciclo.

Un sistema "desequilibrado" se puede manifestar por un insuficiente desarrollo biológico y una escasa disponibilidad de nutrientes, por lo que requeriría de fertilización o coloreado artificial de las balsas para mejorar la producción. Otro problema típico, el exceso de nutrientes, genera un exceso de materia orgánica, que a su vez causa viscosidad en la salmuera y se traduce en cristales huecos o pequeños que podrían retener partículas. Las prácticas de gestión de una salina han de centrarse en mantener las condiciones físico químicas estables en las balsas y el ecosistema en su conjunto en equilibrio y libre de contaminación. Cuando se abandona la producción de sal, el flujo de salmuera se detiene, el equilibrio entre unas balsas y otras desaparece, se uniformizan las condiciones en el conjunto de la salina y las especies halófilas, raras y frágiles, son sustituidas por otras generalistas e incluso oportunistas. Como consecuencia, se banaliza el entramado de redes tróficas secuenciales y se reduce la biodiversidad allí presente. Este desequilibrio, si no es adecuadamente remediado, puede resultar en un emprobrecimiento muy grave de los valores naturales del espacio salinero (Plata \& Erkiaga, 2018).

Así, se concluye que los paisajes de la sal son ambientes extremadamente importantes en términos de biodiversidad. No tanto por el número de especies que allí existen, que, como corresponde a ecosistemas extremos, es generalmente inferior al de otro tipo de ecosistemas, sino porque se trata de especies en muchos casos endémicas $y$, en general, raras $y / o$ de distribución muy restringida. La producción de sal en una salina constituye así no sólo un reservorio de biodiversidad halófila; una isla biogeográfica (sobre todo si se trata de una salina de interior), sino que se puede decir que contribuye al incremento de la biodiversidad a escala local y regional. Es importante entender la biodiversidad como la diversidad de especies, pero también de variabilidad 
genética dentro de las mismas; asunto que mejora a medida que aumenta la abundancia de esa especie.

Pero al tiempo que la producción de sal depende de la salud del ecosistema, éste se beneficia de la producción de sal. No en vano se trata de un espacio antropizado en el que se crean ambientes secuenciales de salinidad y comunidades tróficas específicas, que de otra manera no existirían. De aquí se comprende que la producción de sal es un elemento imprescindible en el ecosistema de una salina, que es, por otra parte, su razón de ser. Las salinas se pueden considerar un tipo especial de ecosistema en el cual la mano humana no sólo debe ser tolerada, sino que es necesaria para contribuir a la conservación y mejora de la biodiversidad al tiempo que se mantiene una actividad económica (Korovessis y Lekkas, 1999, Carrasco \& Hueso, 2012, Petanidou \& Dalaka, 2009). Los valores naturales de las salinas no sólo constituyen un pilar fundamental para su funcionamiento como instalaciones productivas, sino que se ven conservados y mejorados por la propia actividad salinera.

\section{LA SAL COMO PRODUCTO "AGRÍCOLA"}

La sal obtenida de forma artesanal se puede considerar, con todo derecho, como un producto agrícola. Así ha sido reconocido por normativa en países como Francia y Portugal. Podría resultar un poco extraño considerar un compuesto mineral, inorgánico e inerte, la sal, como un producto agrícola. Pero no lo es tanto si en vez del producto se considera el proceso. La sal es químicamente la misma sustancia independientemente de cómo se obtenga. Los métodos de recogida de sal son los que marcan la diferencia en el carácter del producto. Al contrario que en la actividad minera o industrial, la cosecha de sal por evaporación solar requiere un manejo del recurso y de su ambiente que va más allá de su simple recolección (Carrasco, 2017). El referente de producción más cercano en este caso, por ejemplo, son las huertas, pues ambas se basan en un sistema de gestión y aprovechamiento de agua (Quesada, 1995). Esta manera común de manejar el agua tiene su reflejo en las herramientas, en los gestos y la organización del trabajo y en el lenguaje vernáculo (Carrasco, 2017).

Para que una salina de evaporación solar funcione son precisas unas condiciones ambientales específicas. Además de la materia prima, es decir, la salmuera, son necesarios períodos largos y constantes de insolación en determinadas épocas del año. Esenciales son los vientos, que pueden ser de dirección variable, y preferibles las brisas a los vientos fuertes. Las diferencias térmicas entre la noche y el día harán que cristalice de una u otra manera. Una condición imprescindible es la baja humedad relativa del aire que es la que explica la presencia de salinas de evaporación solar en unos lugares y no en otros. La capa freática del suelo debe estar en contacto con la base de las construcciones dedicadas al almacenaje y evaporación de la salmuera, para evitar filtraciones. Por último, pero no menos importante, es necesario un terreno con una superficie suficiente y apropiada, en cuanto a extensión y nivel (Carrasco, 2017). 


\section{LA SAL COMO PRODUCTO GOURMET: EL PAISAJE EN EL PLATO}

Por otro lado, además, es importante reseñar que las sales obtenidas de forma artesanal guardan la huella del salinero. El tamaño, la forma y la solidez de los cristales depende en gran medida del arte del salinero. Y éste, a su vez, conoce su sal como nadie. No hay dos sales artesanales iguales, fruto de una combinación de la composición y del manejo del recurso. La prueba es que durante catas a ciegas desarrolladas en diversas localidades por IPAISAL, Ios salineros locales fueron capaces de reconocer su propia sal.

Las sales no refinadas cosechadas a mano se han ido recuperando o incluso han surgido como un nuevo producto culinario (Drake \& Drake, 2011). En algunos casos, gracias al apoyo de las administraciones públicas y a la financiación, la elaboración artesanal de la sal vuelve a estar en alza y las sales cosechadas a mano son cada vez más apreciadas. En este sentido, son muy meritorios los proyectos financiados por la UE como ALAS, SEL y ECOSAL Atlantis, que han facilitado la consolidación de esta actividad en amplias zonas del Mediterráneo y del Atlántico europeo. Estos proyectos han contribuido a la profesionalización de la actividad salinera artesanal, con cursos de formación, publicaciones, intercambio de experiencias y conocimientos, promoción y, no menos importante, recuperación de infraestructuras de los lugares de producción de sal. Nuevos emprendedores están reconquistando poco a poco salinas abandonadas o estancadas y creando con ellas paisajes de la sal vivos (Hueso 2015, 2019). Del mismo modo, existen numerososn certificados que apoyan la sal artesanal y la sal de calidad (Slow Food, Label Rouge, diversas certificaciones orgánicas) e incuso reconocen y protegen su origen geográfico (Hueso 2019).

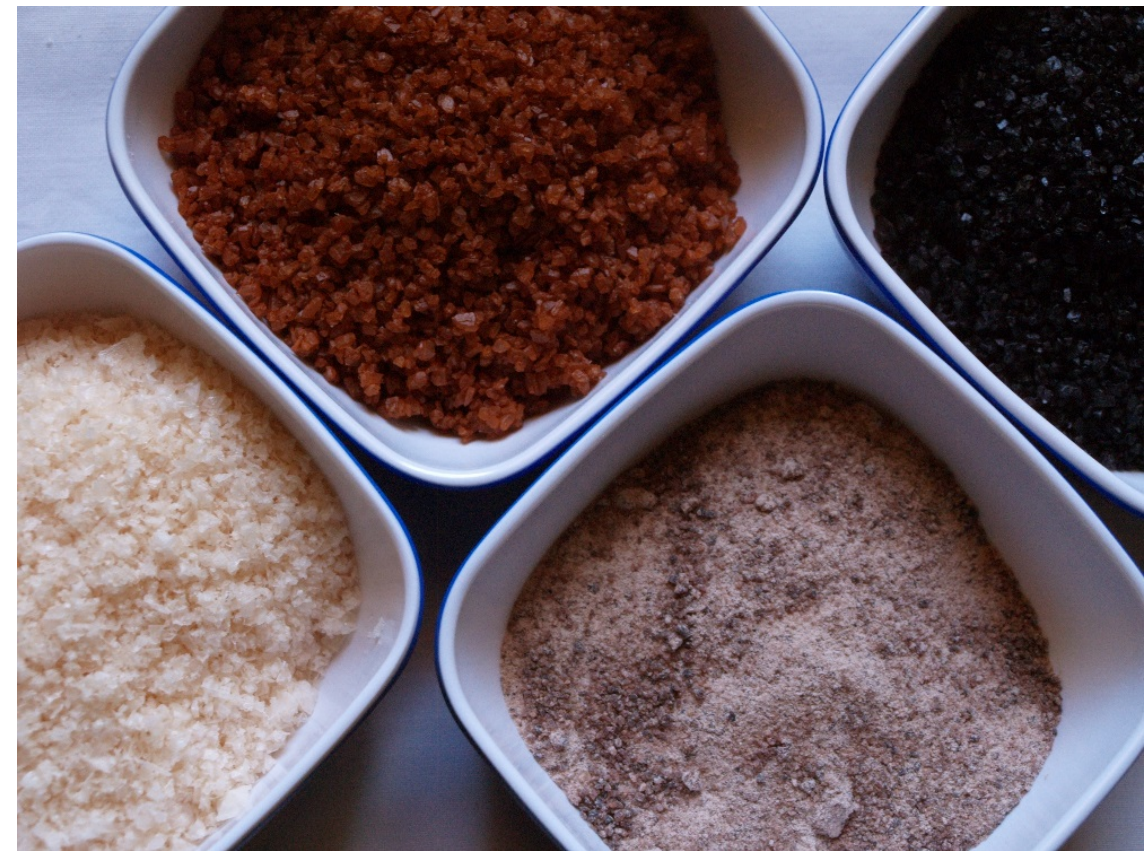

Figura 23: Sales de diferentes colores. La fila de arriba muestra sales coloreados con sustancias naturales (arcilla y carbón activado, respectivamente), mientras que las demás muestran sus tonos habituales Fuente: Katia Hueso / IPAISAL 
Inspiradas por la sal gris francesa, un gran número de sales artesanales aspiran hoy en día a obtener un éxito comercial similar. En el contexto de la popularización de la gastronomía, de un estilo de vida más lento y consciente y de una sensibilidad más profunda hacia los paisajes culturales y sus productos naturales, es evidente que la sal se está convirtiendo en un nuevo alimento a explorar y explotar (Beltran, 2008, Hueso, 2013). Hay abundantes libros de recetas específicas de sal (por ejemplo, Barzini \& Fassone, 2007, BonnavesAguillaume, 2007, Robert, 2007, Aikman-Smith \& Gregson, 2009, Gubler \& Glynn, 2010, Bitterman, 2013), aparecen tiendas de alimentos de calidad especializadas en sal en lugares tan alejados como Barcelona, Ámsterdam y Portland y algunos espacios salineros cuentan con chefs de renombre para promover sus productos, como Salinas de Añana, en Álava (España). Una reciente guía de referencia de sales culinarias publicada por Mark Bitterman (2010) incluye más de 200 sales de este tipo en todo el mundo, demostrando así que no se trata de un fenómeno local ni efímero.

\section{CONCLUSIÓN}

La sal artesanal no es, pues, sólo un producto gourmet. Es el motor más fuerte para la protección de los paisajes de la sal y la recuperación del patrimonio y mejora los medios de vida locales por varias razones. Es la sal que da vida a sus paisajes de referencia. Para cosechar la sal a mano, el sitio debe contar con infraestructuras, dispositivos, herramientas, edificios..., pero también con el conocimiento y la experiencia actualizados de sus trabajadores. Y como se ha visto, se necesita un delicado equilibrio ecológico para producir sal, ya que la biota que se encuentra en las salinas de evaporación solar está íntimamente ligada a los fenómenos biogeoquímicos que tienen lugar en ellas. La vida en la sal es, pues, garantía de vida para los paisajes de la sal.

\section{BIBLIOGRAFÍA}

Aikman-Smith, V. y Gregson, J. (2009). Salt: Cooking with the world's favorite seasoning. Londres, Reino Unido: Ryland Peters \& Small, 64 pp.

Amat, F., Hontoria, F., Navarro, J. C., Vieira, N., y Mura, G. (2007). Biodiversity loss in the genus Artemia in the Western Mediterranean Region. Limnetica 26, (2), 387-404.

Barzini, S. y Fassone, F. (2007). Sale. Un pizzico non vale l'altro. Roma, Italia: Gambero Rosso. 157 pp.

Beltran, O. (2008). El paisaje de la sal, en el plato. A propósito de las sales gourmet y las salinas tradicionales. El Alfolí, 4, 17-23.

Bitterman, M. (2010). Salted: A manifesto on the world's most essential mineral, with recipes. Nueva York, EEUU: Ten Speed Press, 320 pp.

Bitterman, M. (2013). Salt block cooking: 70 Recipes for grilling, chilling, searing, and serving on Himalayan salt blocks. Kansas City, EEUU: Andrew McMeel Publishing, 216 pp. 
Bonnaves-Aguillaume, K. (2007). Les recettes au sel. Roissy-sous-Bois, Francia: Ed. Gisserot

Breckle, S. W. (2002). Salinity, halophytes and salt affected natural ecosystems. En: Läuchli A. y Lüttge U. (eds). Salinity: Environment - Plants - Molecules. Dordrecht, Países Bajos: Springer, pp. 53-77.

Carrasco, J.-F. (2017). Las salinas de interior como actividad agrícola. El Alfolí 20, 13-19.

Carrasco, J. F. y Hueso, K. (2012). La actividad productiva como sostén directo de un paisaje y su biodiversidad: El caso de la producción de sal por evaporación solar. En: Madrid: Actas del Congreso Nacional de Medio Ambiente (CONAMA 2012).

Catry, T., Ramos, J. A., Catry, I., Allen-Revez, M. y Grade, N. (2004). Are salinas a suitable alternative breeding habitat for Little Terns Sterna albifrons?. Ibis, 146(2), 247-257.

Cirujano, S. y Medina, L. (2014). Plantas acuáticas de las lagunas y humedales de Castilla-La Mancha. Toledo, España: Junta de Comunidades de Castila-La Macha.

Comín, F.A. y Alonso, M. (1988). Spanish salt lakes: Their chemistry and biota. Hydrobiologia, 158, 237-245.

Davis, J. S. (1980). Biological management of solar saltworks. En: Coogan, A. H. y Hauber, L. (Eds.) Actas del Fifth Symposium on Salt, Cleveland, Ohio: Northern Ohio Geological Society, pp: 265-268.

Davis, J. S. (2000). Structure, function and management of the biological system for seasonal solar saltworks. Global NEST Journal, 2, 217-226.

Davis, J. S. (2006). Biological and physical management information for commercial solar saltworks. Actas del 1st International Conference on the Ecological Importance of Solar Saltworks (CEISSA 2009), Santorini, pp: 5-14.

Drake, S. L. y Drake, M.A. (2011). Comparison of salty taste and time intensity of sea and land salts from around the world. Journal of Sensory Studies, 26, 2534.

Dyall-Smith, M., Tang, S.-L. y Bath, C. (2003). Halobacterial viruses: how diverse are they? Res. Microbiol., 154, 309-31.

Fensham, F. C. (1962). Salt as Curse in the Old Testament and the Ancient Near East. The Biblical Archaeologist, 25(2), 48-50.

Gómez, R., Hurtado, I., Suárez, M. L. y Vidal-Abarca, M. R. (2005). Ramblas in Southeast Spain: threatened and valuable ecosystems. Aquatic Conservation: Marine and Freshwater Ecosystems, 15, 4, 387- 402. 
Green, A. J., Sánchez, M. I., Amat, F., Figuerola, J., Hontoria, F., Ruiz, O. y Hortas, F. (2005). Dispersal of invasive and native brine shrimps Artemia (Anostraca) via waterbirds. Limnology and oceanography, 50(2), 737-742.

Gubler, F. y Glynn, D. (2010). The salt book. Crows Nest NSW, Australia: Arbon Publishing, $208 \mathrm{pp}$.

Guerrero, M.C. y de Wit, R. (1992). Microbial mats in the inland saline lakes of Spain. Limnética, 8, 197-204.

Hontoria, F. y Amat, F. (1992). Morphological characterization of adult Artemia (Crustacea, Branchiopoda) from different geographical origin. Mediterranean populations. Journal of plankton research, 14(7), 949-959.

Hueso, K. (2013). Salt, much more than an edible stone. El Alfolí, 13, 17-23

Hueso, K. (2015). Artisanal salt and its contribution to the sustainable management of a living saltscape. En: Actas de la Conferencia "Otras maneras de comer", Barcelona: Obervatori de I'Alimentació y Fundación Alicia, pp: 11231145.

Hueso, K. (2019). Salt in our veins. The patrimonialisation processes of artisanal salt and saltscapes in Europe and their contribution to local development. Kaiserslautern, Alemania: Parthenon Verlag, 349 pp.

Hueso, K. y Carrasco, J.-F. (2008). Las salinas de los espacios naturales protegidos de la provincia de Guadalajara. Guadalajara: Association of Friends of Inland Salinas, pp. 23-76.

Korovessis, N. A. y Lekkas, T. D. (1999). Solar saltworks production process evolution - wetland function. En: Actas del Post Conference Symposium SALTWORKS: Preserving Saline Coastal Ecosystems. Samos: Global NEST.

López, E., Aguilera, P. A., Schmitz, M. F., Castro, H., \& Pineda, F. D. (2010). Selection of ecological indicators for the conservation, management and monitoring of Mediterranean coastal salinas. Environmental monitoring and assessment, 166(1-4), 241-256.

Martín, J., Cirujano, S., Moreno, M., Peris, J.B. y Stübing, G. (2003). La vegetación protegida en Castilla - La Mancha. Toledo: Junta de Comunidades de Castilla - La Mancha \& Real Jardín Botánico/C.S.I.C., pp. 265-300.

Millán, A., Moreno, J. L. y Velasco, J. (2002). Estudio faunístico y ecológico de los coleópteros y heterópteros acuáticos y semiacuáticos de la provincia de Albacete. Albacete: Instituto de Estudios Albacetenses.

Montes, C. y Martino, P. (1987). Las lagunas salinas españolas. En: Bases científicas para la protección de los humedales en España. Madrid:Real Academia de Ciencias Exactas, Físicas y Naturales, pp. 95-146. 
Moreno, J. L., Millán, A., Suárez, M. L., Vidal-Abarca, M. R. y Velasco, J. (1997). Aquatic Coleoptera and Heteroptera assemblages in waterbodies from ephemeral coastal streams ("ramblas") of south-eastern Spain. Archiv für Hydrobiologie, 141, 93-107.

Oren, A. y Dubinsky, Z. (1994). On the red coloration of saltern crystallizer ponds. Additional evidence for the coloration of halobacterial pigments. International Journal of Salt Lake Research, 3, 9-13.

Pedrós-Alió, C., Calderón-Paz, J. I., MacLean, M. H., Medina, G., Marrasé, C., Gasol, J. M. y Guixa- Boixareu, N. (2000). The microbial food web along salinity gradients. FEMS Microbiol. Ecol., 32,143-155.

Petanidou, T. y Dalaka, A. (2009). Mediterranean's changing saltscapes: A study of the abandonment of salt-making business in Greece. Global NEST Journal, 11, 4, 415-433.

Plata, A. \& Erkiaga, A. (2018). El sistema de producción de sal de Añana. Valle Salado (Araba, País Vasco). Bilbao, España: Universidad del País Vasco, 170 pp.

Quesada, T. (1995). El agua salada y las salinas. En: El agua en la agricultura de Al-Andalus. Barcelona: Ed. Lunwerg, pp. 57-80.

Robert, J. (2007). Les meilleures recettes de la cuisine au gros sel. Rennes, France: Ed. Ouest France.

Sadoul, N., Walmsley, J. y Charpentier, B. (1998). Salinas and nature conservation. Tour du Valat: MedWet, pp. 71-82.

Sundaresan, S., Ponnuchamy, K. y Rahaman, A. A. (2006). Biological management of Sambhar lake saltworks (Rajasthan, India). En: Actas del 1st International Conference on the Ecological Importance of Solar Saltworks (CEISSA 2009), Santorini, pp: 199-208.

Ventosa, A. y Ventosa, A. (2004). Halophilic microorganisms. Berlin: Springer.

Yi, R., Hongo, Y., Yoda, I., Adam, Z. R., Fahrenbach, A. C. (2018). Radiolytic Synthesis of Cyanogen Chloride, Cyanamide and Simple Sugar Precursors. Chemistry Select, 3, 10169. 